\title{
Integrative medicine models
}

\section{Sofia Raptopoulou ${ }^{1, *}$}

\section{${ }^{1}$ Reflexologist, Athens, Greece. * sofiarapt@gmail.com}

Patients with chronic pain and chronic diseases are looking for ways to combat the health implications, to alleviate the side effects of treatments and to improve their quality of life.

The medical community is constantly looking for ways to provide the comprehensive health care that patients need. In this context, more pluralistic healthcare systems began to develop, combining conventional and complementary approaches in a coordinated way and to varying degrees.

These systems are referred to as Integrative (UK) or Integrated (USA) Medicine.

Two dominant models of Integrative Medicine have been developed.

The first is a selective combination of Complementary Therapies used as add-on to the treatment proposed by conventional medicine, based on evidence from Research and Practice (Supplementary Model).

The second includes only Evidence-Based Complementary Treatments that are integrated in the usual care (Collaborative Model).

These two models have different levels of autonomy, control and responsibility among the participating health professionals. Despite their differences, both models focus on the patient and offer a holistic approach.

In Greece, a Supplementary Model of Integrated Medicine is offered at the Pain Clinic of Aretaieion Hospital. This model selectively combines various Complementary Treatments such as Reflexology, Shiatsu, Acupuncture, Psychological support, etc., as add-on to the conventional treatments thereby maximizing the beneficial results and can serve as a pioneering example for the development of Integrated Medicine systems in Greece.

A well-designed model of Integrated Medicine, combining every possible means and every available treatment and that includes all the necessary safety checks can be particularly effective in achieving the goal of any medical system. The goal of a comprehensive treatment of the disease and its side effects across all aspects of the patient's life. 